\title{
ARTICLE
}

\section{Circulating IL-18 and the risk of type 2 diabetes in women}

\author{
M. F. Hivert • Q. Sun • P. Shrader • C. S. Mantzoros • \\ J. B. Meigs $\cdot$ F. B. Hu
}

Received: 31 March 2009 / Accepted: 19 June 2009 /Published online: 8 August 2009

(C) Springer-Verlag 2009

\begin{abstract}
Aims/hypothesis The pathophysiology of type 2 diabetes involves pro-inflammatory pathways. We tested the hypothesis that IL-18 predicts future diabetes cases.

Methods We used a nested case-control design based in the Nurses' Health Study. Baseline blood samples were collected between 1989 and 1990. Questionnaires to assess body weight, lifestyle (physical activity, diet, smoking) and diabetes diagnosis were sent out and assessed biennially
\end{abstract}

Electronic supplementary material The online version of this article (doi:10.1007/s00125-009-1455-z) contains supplementary material, which is available to authorised users.

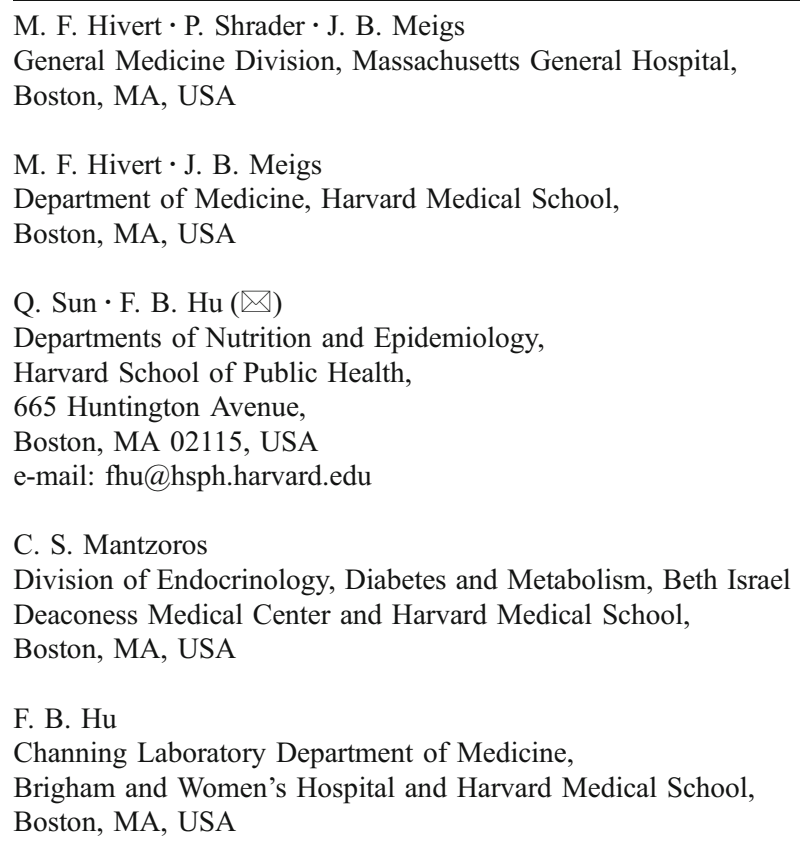

(follow-up until 2002). Cases $(n=1,012)$ were defined as women developing type 2 diabetes at least 1 year after blood sampling. Control women $(n=1,081)$ were matched to cases by age, date of blood draw, fasting status and race. We calculated the RR $(95 \% \mathrm{CI})$ of type 2 diabetes in quintiles of IL-18 using conditional logistic regression with the first quintile as referent; adjustments included matching factors, diabetes risk factors, BMI, adipokine levels (adiponectin, resistin) and inflammatory proteins (C-reactive protein, tumour necrosis factor receptor $2(\mathrm{TNF} \alpha-\mathrm{R} 2)$ and IL-6).

Results Higher IL-18 levels were associated with increased risk of developing diabetes, even after adjustment for matching factors and multiple diabetes risk factors: being in the highest quintile of IL-18 was associated with a RR of 1.75 (1.41-2.18) for diabetes relative to the first quintile $(p<0.0001$ for trend). Significant trends in association were still observed after adjustment for BMI (RR 1.44 [1.151.80 ], $p<0.0001$ for trend) and adiponectin levels (RR 1.28 [1.02-1.60], $p=0.006$ for trend). Further adjustment for inflammatory markers in a sub-sample did not significantly change the results.

Conclusions/interpretation Elevated IL-18 levels are associated with higher risk of diabetes. This association is independent of usual risk factors, including BMI and adipokine levels.

Keywords Adipokines - Adiponectin - Case-control study C-reactive protein $\cdot$ Cytokine $\cdot$ Diabetes incidence . Interleukin-18 $\cdot$ Resistin

$\begin{array}{ll}\text { Abbreviations } \\ \text { CRP } & \text { C-reactive protein } \\ \text { HMW } & \text { High molecular weight } \\ \text { HOMA-IR } & \text { HOMA of insulin resistance }\end{array}$


MET Metabolic equivalent task

TNF $\alpha$-R2 Tumour necrosis factor receptor 2

\section{Introduction}

There is increasing evidence that part of the pathophysiology linking obesity and insulin resistance include proinflammatory pathways [1]. Many proteins have been identified in these inflammatory pathways related specifically to excess adiposity, including the pro-inflammatory molecule IL-18. IL-18 was first described in 1989 as an interferon- $\gamma$ inducing factor [2]. We now know that it has many other functions, including augmentation of cell adhesion molecules, synthesis of nitric oxide, chemokine production, induction of Fas ligand and stimulation of TNF $\alpha$ and IL-6 production [2]. The primary sources of IL-18 are macrophages and dendritic cells, but the IL-18 precursor is produced by epithelial cells throughout the body [2]. Adipocytes have also been shown to produce IL-18 [3], but non-adipocyte cells seem to be the major source of IL-18 in the adipose tissue $[4,5]$. It has been hypothesised that IL-18 is produced by muscles in the insulin-resistant state [6]. It remains to be elucidated if IL-18 is implicated in adiposity-related pro-inflammatory pathways or if its actions are part of another specific pathway.

In cross-sectional studies, IL-18 has been associated with excess adiposity $[7,8]$ and metabolic syndrome $[8,9]$. It has also been associated with insulin resistance measured by HOMA [10, 11], IVGTT [12] or clamps [13]. Cross-sectional studies have also shown that levels of IL-18 are higher in patients with type 2 diabetes $[11,14,15]$. In one prospective cohort, diabetes incidence was associated with higher levels of IL-18 at baseline [16]. This association was adjusted for some of the diabetes risk factors, but not for specific dietary information, which has been shown to influence IL-18 levels [17], or for correlated levels of other adipokines known to be associated with insulin resistance [18] and diabetes risk [19]. The independent contribution of circulating levels of IL-18 with diabetes risk remains unresolved. Using a case-control study nested in the prospective follow-up of the Nurses' Health Study [20], we examined whether IL-18 was associated with development of type 2 diabetes and whether this association was independent of BMI, known diabetes risk factors and adiposity-related biomarkers.

\section{Methods}

Study sample The Nurses' Health Study [20] is a well established cohort that initially enrolled 121,700 female US nurses (30 to 55 years old at baseline) in 1976. From baseline, the participants were asked to fill in health-related questionnaires biennially. In 1989 to 1990, blood samples were provided by 32,826 participants. Women who agreed to provide a blood sample were similar to women who did not in respect of age, BMI, smoking history, physical activity, alcohol consumption and past medical history (hypertension, dyslipidaemia, diabetes or cardiovascular disease). In this analysis, cases were defined as women who were free of diabetes, coronary heart disease, stroke and cancer at the time of blood draw and who developed type 2 diabetes at least 1 year after blood collection until the end of follow-up in June 2002. Control participants were also free of diabetes, coronary heart disease, stroke and cancer at the time of the blood sampling and remained free of diabetes during the same follow-up period. Control participants were matched to cases by age at blood draw ( \pm 1 year), date of blood draw ( \pm 3 months), fasting status ( $\geq 8 \mathrm{~h}$ vs $<8 \mathrm{~h}$ since the last meal) and race. The total sample for this analysis was 1,012 case participants and 1,081 control participants. The study was approved by the institutional review board of Brigham and Women's Hospital, Boston, MA.

Ascertainment of type 2 diabetes The periodic biennial health-related questionnaires were used to screen for cases of type 2 diabetes. Each case was then confirmed by a validated follow-up questionnaire about symptoms, diagnostic laboratory test results and diabetes treatment [20]. Consistent with the criteria of the National Diabetes Data Group, diagnosed cases required: (1) an elevated glucose concentration (fasting plasma glucose $\geq 7.8 \mathrm{mmol} / \mathrm{l}$, random plasma glucose $\geq 11.1 \mathrm{mmol} / 1$ or plasma glucose $\geq 11.1 \mathrm{mmol} / 1$ after an oral glucose load) and at least one symptom related to diabetes (excessive thirst, polyuria, weight loss or hunger); (2) no symptoms, but elevated glucose concentrations on two occasions; and (3) treatment with insulin or oral hypoglycaemic medication. For cases of type 2 diabetes identified after 1998, the cut-off point used for fasting plasma glucose concentrations was lowered to $7.0 \mathrm{mmol} / \mathrm{l}$ according to the American Diabetes Association criteria. Validation of this approach using a sub-sample in the Nurses' Health Study revealed high validity $(98.4 \%$ of self-reported cases were confirmed in the medical records and only $0.5 \%$ of controls were false-negatives) [21].

Assessment of lifestyle and dietary information Body weight was self-reported at the time of blood sampling. BMI was calculated as weight (in $\mathrm{kg}$ ) divided by the squared height (in $\mathrm{m}^{2}$ ). Family history of first-degree relatives was reported in the 1982 and 1988 questionnaires. Dietary intake was calculated using the data in the semiquantitative food frequency questionnaire (average from 
1986 and 1990 questionnaires); values for nutrients were adjusted for total energy intake, using the residual method [22]. The 1988 questionnaire included leisure time physical activity assessment (time per week, intensity) which was used to estimate energy expenditure in metabolic equivalent task (MET) h per week [23]. Information about cigarette smoking, menopausal status and use of hormonal replacement therapy was also taken from the 1988 questionnaire. The validity of self-reported body weight, dietary intake and physical activity levels in the Nurses' Health Study has been previously reported [24].

Laboratory procedures A phlebotomy kit was sent to all the women who agreed to provide blood samples. The samples were returned by overnight mail in a frozen water bottle, processed on arrival and stored in liquid nitrogen $\left(-130^{\circ} \mathrm{C}\right.$ or less) until time of analysis. The plasma samples were analysed in randomly fashioned order (by casecontrol pairs). The laboratory personnel were blind to the case-control status of samples. IL-18 concentration was measured by an ELISA method (MBL, Naka-ku Nagoya, Japan); intra- and inter-assay coefficients of variance were $7.26 \%$ and $7.53 \%$ respectively. Details for the assays used for $\mathrm{C}$-reactive protein (CRP), tumour necrosis factor receptor 2 (TNF $\alpha$-R2), IL-6, high molecular weight (HMW) adiponectin and resistin have been previously reported [19, 25, 26]. We had measurements of HMW adiponectin and resistin from all the women included in this case-control study; measurements of CRP, TNF $\alpha$-R2 and IL-6 were available for sub-samples only (Table 1).

Statistical analysis Baseline characteristics of the case and control participants were compared using $\chi^{2}$ tests (for percentages), Student's $t$ tests (for means) and Wilcoxon's rank-sum tests (for medians). Using the distribution thresholds in control participants, IL-18 levels were divided into quintiles. We used conditional logistic regression to calculate RR of type 2 diabetes with the first quintile as referent. The first model was adjusted for matching factors (age at blood draw $[ \pm 1$ year], date of blood draw [ \pm 3 months], fasting status $[\geq 8 \mathrm{~h}$ vs $<8 \mathrm{~h}$ since the last meal] and race). In a second model, we also adjusted for known diabetes risk factors, i.e. physical activity (quintiles of MET h/week), smoking (never, past, current 1-14 cigarettes per day and current $\geq 15$ cigarettes per day), family history of diabetes (yes/no), hormone replacement therapy (premenopausal, never, past, current and information not available), alcohol consumption (non-drinkers, $0.1-4.9,5-10$ and $>10 \mathrm{~g} /$ day), glycaemia load (quintiles), ratio of polyunsaturated fat to saturated fat (quintiles) and intake of cereal fibre, trans fat, magnesium and total energy (all in quintiles). In the third model, BMI adjustment (by category $<23,23-24.9,25-26.9,27-29.9,30-34.9$ and $\geq 35 \mathrm{~kg} / \mathrm{m}^{2}$ ) was added to all the matching and diabetes risk factors. In subsidiary analysis, we adjusted for adiposity using BMI as a continuous variable and assessed the impact of adjusting for waist circumference as a marker of central adiposity. In additional models, we added major adipokines (HMW adiponectin and resistin) to the BMI-adjusted model. In subsidiary analyses of the fewer participants with inflammatory biomarkers measured, we also tested the BMI-adjusted model adding the other biomarkers (CRP, IL-6, TNF $\alpha-R 2$ ), but we caution that those models should be interpreted in light of the smaller number of women with measurements available. Since glucose levels were not available in this cohort, we adjusted for $\mathrm{HbA}_{1 \mathrm{c}}$ levels in the sub-sample of women in whom it had been measured. We tested the trends across the quintiles of IL-18, using the medians of each quintile as continuous variables in the models. We considered two-sided $p$ values $<0.05$ to be significant. Finally, we tested interactions between levels of IL-18 and major risk factors for diabetes (age, BMI, family history of diabetes). Those models were age- and BMIadjusted (except when specifically stratified for that factor). Interactions with two-sided $p$ values $<0.05$ were considered significant, $p<0.2$ was considered to denote a trend [27]. All statistical analyses were conducted with SAS software 9.1 (SAS Institute, Cary, NC, USA).

\section{Results}

Characteristics of the case and control women at baseline are described in Table 1. Women who developed diabetes were more likely to have a family history of diabetes and less likely to use post-menopausal hormones. They were also less active and had higher mean values of BMI and waist circumference. On dietary assessment, they had greater total daily energy intake and more trans fatty acids, but less fibre, magnesium, caffeine and alcohol. As reported before, women who developed diabetes in the future had more adverse levels of adipokines at baseline, i.e. lower levels of HMW adiponectin, but higher levels of resistin [19]. Case participants had higher levels of all the proinflammatory proteins (TNF $\alpha-\mathrm{R} 2, \mathrm{IL}-6$ and CRP [25]) than the control participants at baseline. Levels of IL-18 were $25 \%$ higher in case participants $(323.2 \mathrm{pg} / \mathrm{ml})$ than in control participants $(264.1 \mathrm{pg} / \mathrm{ml}, p<0.0001)$.

Spearman correlation (Table 2) showed that higher IL-18 levels were moderately associated with higher values of BMI and waist circumference, and weakly associated with higher age and lower physical activity. IL-18 levels were weakly associated with some of the dietary components. 
Table 1 Risk factors for type 2 diabetes, and adipokine and inflammatory biomarker circulating levels in case and control women at baseline
Values are expressed as mean (SD), \% or (for biomarkers) median (interquartile range)

Case and control participants' characteristics were compared using $\chi^{2}$ test (for percentages), $t$ test (for means) or Wilcoxon's rank-sum test (for medians)

${ }^{a} n=$ number in the sample except for: waist circumference 761 control, 621 case participants; physical activity 1,066 control, 1,001 case participants; postmenopausal hormone use 1,072 control, 995 case participants; dietary intake 1,072 control, 1,002 case participants; TNF $\alpha$-R2 598 control, 690 case participants; IL-6 577 control, 667 case participants; and CRP, 584 control, 680 case participants

${ }^{\mathrm{b}}$ Matching factor

\begin{tabular}{|c|c|c|c|}
\hline $\begin{array}{l}\text { Characteristic } \\
n^{\mathrm{a}}\end{array}$ & $\begin{array}{l}\text { Control } \\
1,081\end{array}$ & $\begin{array}{l}\text { Case } \\
1,012\end{array}$ & $p$ value \\
\hline \multicolumn{4}{|l|}{ Demographic and anthropometric } \\
\hline $\operatorname{Age}^{\mathrm{b}}($ years $)$ & $55.8(7.0)$ & $56.1(7.0)$ & 0.41 \\
\hline $\mathrm{BMI}^{\mathrm{b}}\left(\mathrm{kg} / \mathrm{m}^{2}\right)$ & $26.3(6.0)$ & $30.1(5.4)$ & $<0.0001$ \\
\hline Waist circumference $(\mathrm{cm})$ & $78.0(11.7)$ & $87.7(12.0)$ & $<0.0001$ \\
\hline Physical activity (MET/week) & $15.5(25.1)$ & $12.2(15.0)$ & 0.0002 \\
\hline Family history of diabetes (\%) & 22.8 & 45.5 & $<0.0001$ \\
\hline Current smoking (\%) & 12.4 & 13.4 & 0.48 \\
\hline Post-menopausal (\%) & 60.6 & 62.7 & 0.31 \\
\hline Current post-menopausal hormone use (\%) & 36.8 & 28.9 & 0.0001 \\
\hline \multicolumn{4}{|l|}{ Dietary intake } \\
\hline Cereal fibre (g/day) & $4.95(2.58)$ & $4.55(2.18)$ & 0.0001 \\
\hline Glycaemic load & $102.0(17.8)$ & $102.2(16.1)$ & 0.85 \\
\hline Trans fatty acid (g/day) & $2.54(0.83)$ & $2.64(0.83)$ & 0.006 \\
\hline Polyunsaturated:saturated fatty acids ratio & $0.57(0.18)$ & $0.56(0.15)$ & 0.05 \\
\hline Magnesium (mg/day) & $306.2(70.5)$ & $297.1(62.7)$ & 0.002 \\
\hline Caffeine (mg/day) & $256.9(197.0)$ & $236.1(184.1)$ & 0.01 \\
\hline Alcohol (g/day) & $5.62(9.07)$ & $3.40(7.15)$ & $<0.0001$ \\
\hline Total energy intake (kJ/day) & $7,409(1,957)$ & $7,651(2,119)$ & 0.007 \\
\hline \multicolumn{4}{|l|}{ Biomarkers circulating levels } \\
\hline $\mathrm{IL}-18(\mathrm{pg} / \mathrm{ml})$ & $264.1(142.8)$ & $323.2(179.1)$ & $<0.0001$ \\
\hline HMW adiponectin $(\mu \mathrm{g} / \mathrm{ml})$ & $6.62(5.53)$ & $3.59(3.62)$ & $<0.0001$ \\
\hline Resistin (ng/ml) & $15.27(9.85)$ & $16.35(11.16)$ & $<0.0001$ \\
\hline CRP (mg/l) & $1.6(2.7)$ & $3.7(4.2)$ & $<0.0001$ \\
\hline $\mathrm{TNF} \alpha-\mathrm{R} 2(\mathrm{pg} / \mathrm{ml})$ & $2,407.5(887.2)$ & $2,646.5(968.7)$ & $<0.0001$ \\
\hline IL-6 (ng/ml) & $1.92(1.51)$ & $2.39(1.82)$ & $<0.0001$ \\
\hline
\end{tabular}

Circulating IL-18 levels also showed modest associations in the expected directions with the other adipokines and biomarkers, the strongest associations being positive correlations ( $r=0.22$ to $0.30 ; p<0.0001$ for all) with the other pro-inflammatory proteins (TNF $\alpha-\mathrm{R} 2$, IL-6 and CRP).

Multivariate models using quintiles of IL-18 levels at baseline to predict development of type 2 diabetes are presented in Table 3. Higher levels of IL-18 were associated with higher risk of incident diabetes, even after adjustment for all diabetes risk factors (Model 2: multivariate RR). Thus being in the highest quintile of IL-18 was associated with a RR (95\% CI) of 1.75 (1.41-2.18) for developing diabetes in the future (relative to the first quintile; $p<0.0001$ for trend). After further adjustment for BMI (Model 3), the RR was reduced (1.44 [1.15-1.80]), but remained significant. Using BMI as a continuous variable instead of in categories, the RR was 1.56 (1.25-1.95) (for the highest compared with the lowest quintile; $p<0.0001$ for trend); this subsidiary analysis confirmed that it is more appropriate to use categorical BMI adjustment. Using waist circumference instead of BMI resulted in the same trend $(p=0.002)$. Adding HMW adiponectin to Model 3 further reduced the RRs, as seen in Model 4 (Table 3); the trend remained significant ( $p=0.006$ for trend). Adding resistin to Model 3 did not change the association of IL-18 with diabetes (see Model 5); adding resistin to Model 4 (including HMW-adiponectin) had no effect on the RRs and resulted in exactly the same trend and $p$ value (not shown). In subsidiary analyses (see Electronic supplementary material [ESM] Table 1), adjustment for other proinflammatory proteins (TNF $\alpha-\mathrm{R} 2$, IL-6 or CRP) did not have a substantial impact on the initial BMI-adjusted model (Model 3), and all trends for the IL-18 association with diabetes remained significant ( $p<0.05$ for trends, all). Even when all the biomarkers (HMW adiponectin, resistin, CRP, IL-6, TNF $\alpha-\mathrm{R} 2$ ) were included in the BMI-adjusted model, the trend for IL-18 quintiles to predict diabetes remained significant ( $p=0.03$ for trend) (ESM Table 1). The subsidiary analysis in the sub-sample for which $\mathrm{HbA}_{1 \mathrm{c}}$ measurements were available (cases $n=402$, controls $n=403$ ) showed similar trends, even after adjustment for this marker of chronic glycaemic exposure. High levels of IL-18 seemed to increase the risk of developing diabetes, regardless of levels of adiponectin, resistin or CRP (Fig. 1).

We conducted interaction analyses with major risk factors (age, BMI and family history of diabetes) for diabetes. The 
Table 2 Spearman correlations of IL-18 with risk factors, adipokines and inflammatory biomarkers in case and controls women at baseline

\begin{tabular}{lcc}
\hline Characteristic & Spearman correlation & $p$ value \\
\hline Demographic and anthropometric & & 0.0008 \\
Age (years) & 0.07 & $<0.0001$ \\
BMI $\left(\mathrm{kg} / \mathrm{m}^{2}\right.$ ) & 0.22 & $<0.0001$ \\
Waist circumference (cm) & 0.20 & 0.005 \\
Physical activity (MET/week) & -0.06 & \\
Dietary intake & & 0.02 \\
Cereal fibre (g/day) & -0.05 & 0.44 \\
Glycaemic load & -0.02 & 0.04 \\
Trans fatty acid (g/day) & 0.04 & 0.009 \\
Polyunsaturated:saturated fatty acids ratio & -0.06 & 0.007 \\
Magnesium (mg/day) & -0.06 & 0.23 \\
Caffeine (mg/day) & -0.03 & 0.002 \\
Alcohol (g/day) & -0.07 & 0.01 \\
Total energy intake (kJ/day) & 0.06 & $<0.0001$ \\
Biomarkers circulating levels & & $<0.0001$ \\
HMW adiponectin $(\mu \mathrm{g} / \mathrm{ml})$ & -0.20 & $<0.0001$ \\
Resistin (ng/ml) & 0.18 & $<0.0001$ \\
CRP (mg/l) & 0.30 & $<0.0001$ \\
TNF $\alpha$-R2 (pg/ml) & 0.30 & \\
IL-6 (ng/ml) & 0.22 & \\
\hline
\end{tabular}

women with a BMI lower than $25 \mathrm{~kg} / \mathrm{m}^{2}$ seemed to have a stronger association of IL-18 with diabetes incidence (RR 2.12 [1.40-3.20] for highest quintile compared with first quintile) than overweight and obese women (RR 1.35 [1.05-
1.74] for the highest quintile compared with first quintile; $p=0.05$ for interaction). We also observed an interesting trend: women without a family history of diabetes seemed to have a stronger association of IL-18 with future diabetes (RR

Table 3 Relative risk, by quintile of IL-18 levels (95\% CI), of developing type 2 diabetes

\begin{tabular}{|c|c|c|c|c|c|c|}
\hline \multirow[t]{2}{*}{ Variable } & \multicolumn{5}{|c|}{ Quintile of IL-18 concentration ${ }^{\mathrm{a}}$} & \multirow[t]{2}{*}{$p$ value for trend } \\
\hline & 1 (lowest) & 2 & 3 & 4 & 5 (highest) & \\
\hline Median (range) & $\begin{array}{l}161.4 \\
(78.4-191.6)\end{array}$ & $\begin{array}{l}215.0 \\
(191.6-239.6)\end{array}$ & $\begin{array}{l}263.8 \\
(239.7-290.9)\end{array}$ & $\begin{array}{l}326.5 \\
(291.1-372.2)\end{array}$ & $\begin{array}{l}451.8 \\
(372.4-3,267.6)\end{array}$ & \\
\hline Cases, $n$ & 108 & 140 & 155 & 239 & 370 & \\
\hline Controls, $n$ & 216 & 216 & 217 & 216 & 216 & \\
\hline \multicolumn{7}{|l|}{ Models, RR (95\% CI) } \\
\hline 1: crude $^{b}$ & Referent & $1.18(0.92-1.52)$ & $1.26(0.98-1.61)$ & $1.58(1.26-1.99)$ & $1.90(1.54-2.36)$ & $<0.0001$ \\
\hline 2: multivariate $\mathrm{RR}^{\mathrm{c}}$ & Referent & $1.20(0.94-1.55)$ & $1.24(0.97-1.59)$ & $1.54(1.22-1.93)$ & $1.75(1.41-2.18)$ & $<0.0001$ \\
\hline 3: multivariate $\mathrm{RR}^{\mathrm{d}}$ & Referent & $1.08(0.84-1.39)$ & $1.08(0.84-1.38)$ & $1.27(1.00-1.60)$ & $1.44(1.15-1.80)$ & $<0.0001$ \\
\hline 4: multivariate $\mathrm{RR}^{\mathrm{e}}$ & Referent & $1.04(0.80-1.33)$ & $1.01(0.78-1.29)$ & $1.16(0.92-1.47)$ & $1.28(1.02-1.60)$ & 0.005 \\
\hline 5: multivariate $\mathrm{RR}^{\mathrm{f}}$ & Referent & $1.08(0.84-1.40)$ & $1.08(0.84-1.39)$ & $1.27(1.00-1.60)$ & $1.44(1.15-1.80)$ & $<0.0001$ \\
\hline
\end{tabular}

${ }^{\mathrm{a}}$ Quintiles based on control distribution

${ }^{\mathrm{b}}$ Adjusted for matching factors (age, date of blood draw, fasting status and ethnicity)

${ }^{\mathrm{c}}$ Also adjusted for physical activity (quintiles of MET/week), smoking (never, past, current 1-14 cigarettes/day and current $\geq 15$ cigarettes/day), family history of diabetes (yes/no), hormone replacement therapy (premenopausal, never, past, current and information not available), alcohol consumption (non-drinkers, $0.1-4.9,5-10$ and $>10 \mathrm{~g} /$ day), glycaemic load (quintiles), ratio of polyunsaturated : saturated fat (quintiles) and intake (quintiles) of cereal fibre, trans fat, magnesium and total energy

${ }^{\mathrm{d}}$ Also adjusted for BMI $\left(<23,23-24.9,25-26.9,27-29.9,30-34.9\right.$ and $\left.\geq 35 \mathrm{~kg} / \mathrm{m}^{2}\right)$

${ }^{\mathrm{e}}$ Model 3 also adjusted for HMW adiponectin

${ }^{\mathrm{f}}$ Model 3 also adjusted for resistin 

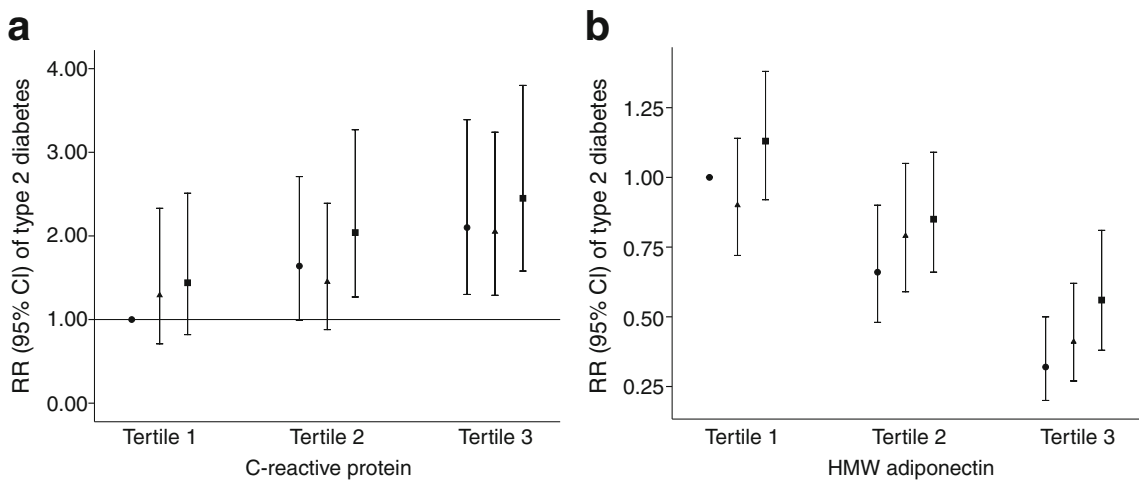

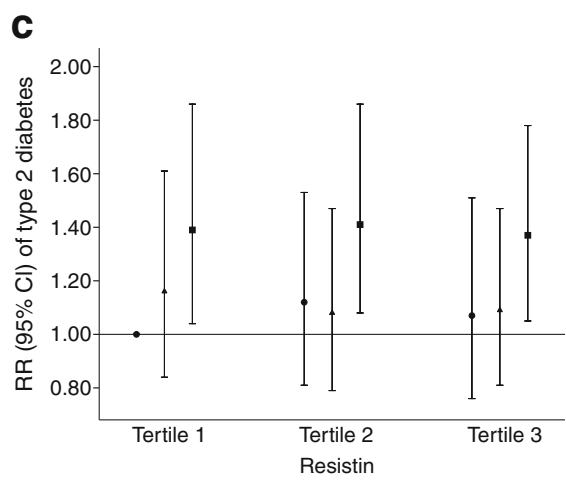

Fig. 1 Multivariate-adjusted RR $(95 \%$ CI) of developing type 2 diabetes, by joint analysis of IL-18 and (a) CRP, (b) HMW adiponectin or (c) resistin. Results are stratified by tertiles. RRs are adjusted for matching factors, diabetes risk factors and BMI. Tertiles of IL-18 are based on control group distribution, i.e.: first tertile, median 182.9 (78.4-226.2) (black circles); second tertile, median
264.5 (226.3-313.0) (black triangles); and third tertile, median 391.5 (313.1-2100.6) (black squares). Tertiles of adipokines were also based on control participants: HMW adiponectin $\leq 4.96 \mu \mathrm{g} / \mathrm{ml}, 4.97-$ $8.58 \mu \mathrm{g} / \mathrm{ml}$ and $>8.58 \mu \mathrm{g} / \mathrm{ml}$; resistin $\leq 12.58 \mathrm{ng} / \mathrm{ml}, 12.59-18.39 \mathrm{ng} /$ $\mathrm{ml}$ and $>18.39 \mathrm{ng} / \mathrm{ml}$; and CRP $\leq 0.95 \mathrm{mg} / \mathrm{l}, 0.96-2.62 \mathrm{mg} / \mathrm{l}$ and $>2.62 \mathrm{mg} / \mathrm{l}$
1.61 [1.18-2.21] for highest quintile compared with first quintile) than women with a positive familial history (RR 1.34 [0.99-1.82] for highest quintile compared with first quintile; $p=0.06$ for interaction).

\section{Discussion}

We have shown that higher circulating plasma levels of IL-18 are associated with future diabetes incidence in a healthy cohort of middle-aged women. This association was independent of well-known risk factors for diabetes, including obesity and dietary intake, and novel risk factors, including levels of adipokines. Interaction analyses suggested that the association of IL-18 with incident diabetes may be stronger in women otherwise considered at low risk of diabetes (such as without family history of diabetes or with lower BMI). This could mean that IL-18 may be implicated very early in the disease process, its predicting effect being dampened by common risk factors; or, in another scheme, it could indicate that IL-18 is part of a pathway different from the one implicated by the usual risk factors.

The role of IL-18 in the development of diabetes is still unclear. It has been shown that adipocytes from obese individuals produce higher levels of IL-18 than those from lean individuals [3]. Other investigators have shown that non-adipocyte cells are the primary source of IL-18 in the adipose tissue of obese individuals [4, 5]. Leick et al. confirmed that obese individuals had higher adipose tissue IL18 mRNA levels than lean individuals and showed that an 8-week exercise programme decreased IL18 mRNA levels in adipose tissue of obese individuals [29]. In contrast, Krogh-Madsen et al. have suggested that adipose tissue is unlikely to be a major source of IL-18; thus in vivo, $\mathrm{TNF} \alpha$ infusion induced insulin resistance in healthy humans (measured by clamps) and increased IL18 mRNA levels in muscles but not in adipose tissue [6]. Non-adipose sources of IL-18 might explain some of the inconsistencies in the literature regarding circulating IL-18 and anthropometric measurements. We and others $[7,8,13]$ have shown a positive association between BMI and IL-18 levels, while other groups have not observed this correlation $[11,16,30]$. BMI is an imperfect proxy for excess adiposity and represents both fat and fat-free mass. Interestingly, in nondiabetic men, IL-18 was not associated with BMI $(r=0.12$, $p=0.1)$, but showed a moderate correlation $(r=0.19$, $p=0.03$ ) with fat-free mass [12], which could support the hypothesis that muscles contribute to circulating IL-18.

Another argument in favour of muscles being the source of IL-18 is that increased adiposity seems to mask the association of IL-18 and the risk of developing diabetes. This hypothesis is supported by our data showing that in women with lower BMI, the association between IL-18 and diabetes tended to be stronger ( $p=0.05$ for interaction). This stronger association for individuals with lower BMI was also suggested in a report on IL-18 levels and diabetes incidence in the MONICA/KORA study [16]. Hung et al. also found a significant interaction $(p=0.03)$ between BMI and an IL-18 association with metabolic syndrome [8]. In a 15 -week weight-loss intervention in obese individuals, Bruun et al. demonstrated that a change in IL-18 levels during the intervention was correlated with changes in HOMA of insulin resistance (HOMA-IR) and not with changes in BMI [10]. The same study also demonstrated that IL18 mRNA or changes in mRNA in subcutaneous adipose tissue were not associated with circulating IL-18, BMI or HOMA-IR at baseline (or with changes over intervention), supporting the hypothesis that the major source of circulating IL-18 in humans is not adipocytes. 
Nevertheless, IL-18 circulating levels have been very consistently associated with insulin resistance [10-13] in accordance with our findings.

If IL-18 is closely implicated in the pro-inflammatory cascade inside the adipose tissue, we would expect its predicting effect to be stronger with adverse levels of adipokines such as low adiponectin. Low levels of circulating adiponectin (both total and HMW forms) have been consistently shown to predict higher risk of developing diabetes, and this was confirmed in the Nurses' Health Study by a previous report [19]. In the present report, we did not observe a stronger effect of IL-18 at more adverse adipokines levels: in each tertile of adiponectin (or resistin), higher levels of IL-18 were associated with an increased risk of developing diabetes. Once again, this suggests that IL-18 might be implicated in a very specific pathway outside adipose tissue. Moreover, our finding that IL-18 is associated with risk of developing diabetes independently of adiponectin levels is concordant with other findings in the literature. For example, Straczkowski et al., using the gold-standard euglycaemic-hyperinsulinaemic clamp to measure insulin sensitivity ( $M$ value), demonstrated that circulating IL-18 was associated with $M$ value, even when adjusted for all the co-variates, including adiponectin [13].

Strengths and limitations Our study has many strengths. Thus we used a well-known cohort of women in a nested case-control design, providing large sample size, prospective follow-up of cases of diabetes, excellent validation of diabetes diagnosis and measurements of an extensive list of risk factors (including detailed information on diet and lifestyle), as well as of biomarkers associated with diabetes, all of which we could adjust for in the models. We used single measurement of biomarkers at baseline, but this has been shown to be reliable compared with more than one measurement over time [31, 32]. We had large numbers of participants, allowing us to use quintiles of IL-18 to test the main association with multiple adjustments in a nonparametric framework. However, our power to detect interactions might have been limited. Our cohort is composed of women predominantly of European origin, so our results might not be generalisable to other ethnic backgrounds. Finally, we adjusted for known confounding factors, but residual confounding cannot be excluded. Since glucose levels are associated with IL-18 levels or diabetes incidence in other cohorts, we cannot exclude the possibility that adjusting for glucose in our multivariate models could have reduced the observed effect, since glucose levels are strong predictors of diabetes; we addressed this limitation by adjusting for $\mathrm{HbA}_{1 \mathrm{c}}$ in the sub-sample for whom measurements were available; this reduced the effect, but did not eliminate the trend. We acknowledge that the absence of glucose measurement in this cohort limits our ability to account for this major confounding factor and that adjusting for $\mathrm{HbA}_{1 \mathrm{c}}$ only partially addresses this limitation. It is possible that factors related to IL-18 at baseline (e.g. obesity) increased the risk of being diagnosed with diabetes. However, adjustment for baseline BMI did not abolish the observed association. Also, stratified analyses by baseline BMI indicated that the association between IL-18 and diabetes was stronger in normal-weight than in overweight and obese women ( $p=0.05$ for interaction).

Conclusion We have shown that high levels of IL-18 are associated with an increased risk of future diabetes, and that this is independent of obesity, other known diabetes risk factors (including dietary aspects), levels of adipokines and other pro-inflammatory proteins. IL-18 might be a stronger predictor of diabetes in women otherwise considered at lower risk of diabetes. Type 2 diabetes is a very heterogeneous disease. It remains to be elucidated whether IL-18 is involved in a very specific pathway that is different from the ones driven by the usual risk factors or whether it is a very early marker of diabetes risk. Our observations, along with other reports, suggest that IL-18 might be involved in non-adipocyte-related inflammatory pathways. Physiological studies and cohort studies with repeated prospective measurements of adipokines and biomarkers are needed to reveal this aspect of the pathophysiology of diabetes.

Acknowledgements The data used in this report were taken from the Nurses' Health Study, which is located in the Channing Laboratory, Department of Medicine, Brigham and Women's Hospital and Harvard Medical School. The National Institutes of Health provided funding for this study (NIH grant DK58845). The funding source played no role in the collection, analysis or interpretation of the data, or the decision to submit the manuscript for publication. M. F. Hivert was supported by the Centre de Recherche Medicale de l'Universite de Sherbrooke (CRMUS) and a Canadian Institute of Health Research (CHIR) Fellowships Health Professional Award. J. B. Meigs was supported by a Career Development Award from the American Diabetes Association and by NIDDK K24 DK080140, and currently has research grants from GlaxoSmithKline and sanofiaventis, as well as consulting agreements with sanofi-aventis, GlaxoSmithKline, Interleukin Genetics, Kalypsis and Outcomes Science. C. S. Mantzoros is supported by NIH grants DK58785, DK79929, DK 081913 and DK58845, and by a discretionary grant from Beth Israel Deaconess Medical Center. The authors would like to thank J. Chamberland for his contribution to the IL-18 assays in C. S. Mantzoros's laboratory.

Duality of interest The authors declare that there is no duality of interest associated with this manuscript.

\section{References}

1. Yudkin JS (2003) Adipose tissue, insulin action and vascular disease: inflammatory signals. Int J Obes Relat Metab Disord 27 (Suppl 3):S25-S28 
2. Dinarello CA (2006) Interleukin 1 and interleukin 18 as mediators of inflammation and the aging process. Am J Clin Nutr $83: 447 \mathrm{~S}-455 \mathrm{~S}$

3. Skurk T, Kolb H, Muller-Scholze S et al (2005) The proatherogenic cytokine interleukin-18 is secreted by human adipocytes. Eur J Endocrinol 152:863-868

4. Fain JN, Tichansky DS, Madan AK (2006) Most of the interleukin 1 receptor antagonist, cathepsin $\mathrm{S}$, macrophage migration inhibitory factor, nerve growth factor, and interleukin 18 release by explants of human adipose tissue is by the non-fat cells, not by the adipocytes. Metab Clin Exp 55:1113-1121

5. Wood IS, Wang B, Jenkins JR et al (2005) The pro-inflammatory cytokine IL-18 is expressed in human adipose tissue and strongly upregulated by TNFalpha in human adipocytes. Biochem Biophys Res Commun 337:422-429

6. Krogh-Madsen R, Plomgaard P, Moller K et al (2006) Influence of TNF-alpha and IL-6 infusions on insulin sensitivity and expression of IL-18 in humans. Am J Physiol: Endocrinol Metab 291: E108-E114

7. Hulthe J, McPheat W, Samnegard A et al (2006) Plasma interleukin (IL)-18 concentrations is elevated in patients with previous myocardial infarction and related to severity of coronary atherosclerosis independently of C-reactive protein and IL-6. Atherosclerosis 188:450-454

8. Hung J, McQuillan BM, Chapman CM et al (2005) Elevated interleukin-18 levels are associated with the metabolic syndrome independent of obesity and insulin resistance. Arterioscler Thromb Vasc Biol 25:1268-1273

9. Van Guilder GP, Hoetzer GL, Greiner JJ et al (2006) Influence of metabolic syndrome on biomarkers of oxidative stress and inflammation in obese adults. Obesity 14:2127-2131

10. Bruun JM, Stallknecht B, Helge JW et al (2007) Interleukin-18 in plasma and adipose tissue: effects of obesity, insulin resistance, and weight loss. Eur J Endocrinol 157:465-471

11. Fischer CP, Perstrup LB, Berntsen A et al (2005) Elevated plasma interleukin-18 is a marker of insulin-resistance in type 2 diabetic and non-diabetic humans. Clin Immunol 117:152-160

12. Bosch M, Lopez-Bermejo A, Vendrell J, Musri M, Ricart W, Fernandez-Real JM (2005) Circulating IL-18 concentration is associated with insulin sensitivity and glucose tolerance through increased fat-free mass. Diabetologia 48:1841-1843

13. Straczkowski M, Kowalska I, Nikolajuk A et al (2007) Increased serum interleukin-18 concentration is associated with hypoadiponectinemia in obesity, independently of insulin resistance. Int $\mathrm{J}$ Obes 31:221-225

14. Aso Y, Okumura K, Takebayashi K et al (2003) Relationships of plasma interleukin-18 concentrations to hyperhomocysteinemia and carotid intimal-media wall thickness in patients with type 2 diabetes. Diabetes Care 26:2622-2627

15. Esposito K, Nappo F, Giugliano F et al (2003) Cytokine milieu tends toward inflammation in type 2 diabetes. Diabetes Care $26: 1647$
16. Thorand B, Kolb H, Baumert J et al (2005) Elevated levels of interleukin-18 predict the development of type 2 diabetes: results from the MONICA/KORA Augsburg Study, 1984-2002. Diabetes 54:2932-2938

17. Esposito K, Nappo F, Giugliano F et al (2003) Meal modulation of circulating interleukin 18 and adiponectin concentrations in healthy subjects and in patients with type 2 diabetes mellitus. Am J Clin Nutr 78:1135-1140

18. Hivert MF, Sullivan LM, Fox CS et al (2008) Associations of adiponectin, resistin, and tumor necrosis factor-alpha with insulin resistance. J Clin Endocrinol Metab 93:3165-3172

19. Heidemann C, Sun Q, van Dam RM et al (2008) Total and highmolecular-weight adiponectin and resistin in relation to the risk for type 2 diabetes in women. Ann Intern Med 149:307-316

20. Hu FB, Manson JE, Stampfer MJ et al (2001) Diet, lifestyle, and the risk of type 2 diabetes mellitus in women. $\mathrm{N}$ Engl J Med 345:790-797

21. Field AE, Coakley EH, Must A et al (2001) Impact of overweight on the risk of developing common chronic diseases during a 10year period. Arch Intern Med 161:1581-1586

22. Willett WC, Howe GR, Kushi LH (1997) Adjustment for total energy intake in epidemiologic studies. Am J Clin Nutr 65:1220S-1231S

23. Hu FB, Sigal RJ, Rich-Edwards JW et al (1999) Walking compared with vigorous physical activity and risk of type 2 diabetes in women: a prospective study. JAMA 282:1433-1439

24. Colditz GA, Hankinson SE (2005) The nurses' health study: lifestyle and health among women. Nat Rev Cancer 5:388-396

25. Hu FB, Meigs JB, Li TY, Rifai N, Manson JE (2004) Inflammatory markers and risk of developing type 2 diabetes in women. Diabetes 53:693-700

26. Schulze MB, Solomon CG, Rifai N et al (2005) Hyperproinsulinaemia and risk of type 2 diabetes mellitus in women. Diabet Med 22:1178-1184

27. Selvin S (1991) Statistical analysis of epidemiologic data. Oxford University Press, New York

28. Hu FB, Meigs JB, Li TY et al (2004) Inflammatory markers and risk of developing type 2 diabetes in women. Diabetes 53:693-700

29. Leick L, Lindegaard B, Stensvold D et al (2007) Adipose tissue interleukin-18 mRNA and plasma interleukin-18: effect of obesity and exercise. Obesity 15:356-363

30. Vilarrasa N, Vendrell J, Maravall J et al (2006) IL-18: relationship with anthropometry, body composition parameters, leptin and arterial hypertension. Horm Metab Res 38:507-512

31. Kaplan RC, Ho GY, Xue X et al (2007) Within-individual stability of obesity-related biomarkers among women. Cancer Epidemiol Biomark Prev 16:1291-1293

32. Lee SA, Kallianpur A, Xiang YB et al (2007) Intra-individual variation of plasma adipokine levels and utility of single measurement of these biomarkers in population-based studies. Cancer Epidemiol Biomark Prev 16:2464-2470 